\title{
O USO DE VÍDEOS E HISTÓRIAS DIGITAIS COMO RECURSOS DIGITAIS NA SALA DE AULA DE INGLÊS COMO LÍNGUA ESTRANGEIRA
}

\author{
The use of videos and digital stories as digital resources in the English \\ as a foreign language classroom
}

\author{
Caroline Chioquetta LORENSET \\ Instituto Federal de Santa Catarina \\ caroline.lorenset@ifsc.edu.br \\ https://orcid.org/0000-0002-1999-0134 \\ Celso Henrique Soufen TUMOLO \\ Universidade Federal de Santa Catarina \\ celso.tumolo@ufsc.br \\ http://orcid.org/0000-0001-5045-8712 \\ Marinho Cristiel BENDER \\ Programa de Pós-Graduação em Inglês \\ Universidade Federal de Santa Catarina \\ marinhobender@gmail.com \\ https://orcid.org/0000-0002-9029-836X
}

RESUMO: Recursos digitais podem auxiliar o processo de ensino de línguas de forma inovadora e seu uso tem se tornado cada vez mais frequente em práticas didático-pedagógicas. Este artigo busca trazer e aprofundar reflexões gerais sobre recursos digitais e seu uso inovador como auxiliares para o ensino e desenvolvimento de inglês como língua estrangeira. Discutimos aporte teórico devidamente embasado e caracterizamos o uso de tecnologias digitais como vídeos e histórias digitais. Desta maneira, o objetivo é difundir e discutir o uso destes recursos digitais na sala de aula de inglês como língua estrangeira, trazendo reflexões sobre aspectos essenciais para o desenvolvimento de línguas estrangeiras. Ao apontar e exemplificar como os diversos recursos digitais podem ser usados, espera-se contribuir para sua incorporação na prática docente de professores na área de ensino e desenvolvimento de línguas estrangeiras. PALAVRAS-CHAVE: Recursos Digitais; Ensino e Desenvolvimento de Inglês como Língua Estrangeira; Vídeos; Histórias Digitais.

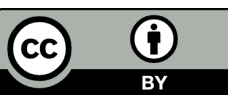




\begin{abstract}
Digital resources can help the language teaching process in an innovative way and its use has become increasingly frequent in classroom practices. The present article seeks to deepen general reflections regarding digital resources and their innovative use as aids for teaching and developing English as a foreign language. We discuss a properly grounded theoretical approach and characterize the use of digital resources such as videos and digital stories. In this way, the objective is to disseminate and discuss the use of digital resources in the classroom of English as a foreign language, bringing reflections on essential aspects for the development of foreign languages. By pointing out and exemplifying how the digital resources can be used, it is expected to contribute to their use and application into the teaching practice of teachers in the field of teaching and developing foreign languages. KEYWORDS: Digital Resources; Teaching and Developing English as a Foreign Language; Videos; Digital Stories.
\end{abstract}

\title{
INTRODUÇÃO
}

Muitos recursos tecnológicos têm sido utilizados nos contextos educacionais através dos anos, desde livros didáticos bem ilustrados, quadro-negro e quadro branco, fita cassete e videocassete, CDs, (TUMOLO, 2017, 2014) entre outros, e também plataformas digitais mais recentes, como tablets e smartphones. Além disso, muitas têm sido as estratégias de ensino e aprendizagem usadas com os recursos tecnológicos disponíveis a fim de proporcionar um processo de desenvolvimento do conhecimento mais significativo e eficaz para os aprendizes. A combinação de recursos tecnológicos com adequadas estratégias pode trazer ótimo resultado para a sala de aula de língua estrangeira (doravante $\mathrm{LE}^{1}$ ), podendo resultar no desenvolvimento ${ }^{2}$ contextualizado e motivador. Este artigo busca trazer uma reflexão sobre o uso de recursos digitais como o a) vídeo como recurso multimidiático para o desenvolvimento da língua inglesa, e b) de histórias digitais para o desenvolvimento em especial, da pronúncia e da escrita, para que possam ser incluídos em contextos de sala de aula de ensino e desenvolvimento de inglês como LE.

\footnotetext{
${ }^{1}$ Neste artigo, o conceito Língua Estrangeira (LE) é usado referente à língua que é ensinada essencialmente em contextos formais (pedagógicos) em que, tipicamente, há pouca exposição ou baixa frequência de exposição a ela por parte dos aprendizes.

${ }^{2}$ Desenvolvimento de uma LE está relacionado ao desenvolvimento das quatro habilidades e dos conhecimentos sintático (gramatical) e lexical (vocabular), que são essenciais ao conhecimento da língua assim como as habilidades necessários para se fazer uso desses conhecimentos. Ademais, o desenvolvimento da língua se dá por meio de uso receptivo (input) e produtivo (output) em contextos variados.
}

Revista X, v. 16, n. 3, p. 728-741, 2021. 


\section{VÍDEO COMO RECURSO MULTIMIDIÁTICO}

Materiais multimídia podem ser definidos como materiais que fazem uso da combinação de imagens e palavras para distribuir informações. Conduzindo esta definição para o contexto educacional, eles podem ser definidos como materiais que fazem uso de imagens e palavras, ou seja, de informação visual acompanhada de informação verbal para a transmissão de informação em um contexto de aprendizagem (MAYER, 2005a; 2009). Considerando que as informações sejam disponibilizadas por meio de imagens e palavras - visual e verbal - é possível definir vídeos como um recurso multimídia que pode ser incorporado no contexto educacional, mais especificamente, no contexto de ensino/desenvolvimento de inglês como LE.

A incorporação de vídeos pode trazer vários benefícios para o complexo processo de ensinar e desenvolver conceitos e aspectos linguísticos. Não somente os aprendizes se tornam mais ativos no processo de construção de seu próprio conhecimento, trazendo experiências e aprendizados anteriores, mas também, estes materiais acarretam em oportunidades de compartilhar os conhecimentos em blogs ou em mídias sociais, por exemplo, aumentando a sua autonomia e agência. Vídeos oferecem excelentes oportunidades no processo de desenvolvimento da língua, uma vez que eles trazem input diversificado e formas variadas de abordagem, resultando em um processo de desenvolvimento significativo e eficaz (MAYER, 2009). Além disso, eles podem ter um papel importante no contexto do ensino e aprendizagem de uma LE, expondo os aprendizes à combinação de input linguístico (verbal) e visual (imagético) (PLASS; JONES, 2005) que, por sua vez é essencial para que o que abstrato se torne mais tangível e facilite o processo de produção da linguagem-alvo, ou seja output.

No caso de input verbal, este pode ser apresentado em vídeos de duas formas: oral e escrito. O input oral pode ser apresentado em forma de diálogos, como em situações conversacionais, ou em forma de narração, que é o caso de tutoriais ou documentários. Já no caso de input escrito, a linguagem-alvo é apresentada em forma de legendas.

Embora a linguagem oral seja sempre apresentada na linguagem-alvo sendo desenvolvida, ou seja, na LE dos aprendizes, quando vídeos apresentam legendas, estas podem ser categorizadas de duas formas: legendas interlinguísticas e legendas intralinguísticas (LAVAUR; BAIRSTOW, 2011; DANAN, 2004). As legendas interlinguísticas referem-se à inserção de informação verbal nos vídeos que são diferentes da linguagem falada. Por exemplo, em um vídeo o narrador usa o inglês na forma oral (LE do aprendiz), e a legenda é apresentada em Português (língua materna do aprendiz). 
Já as legendas intralinguísticas, apresentam-se no mesmo idioma em que a linguagem oral do vídeo. Por exemplo, um vídeo apresenta a fala do narrador e as legendas em inglês (LE dos aprendizes). A inserção de legendas em vídeos pode exercer a função de auxiliar os aprendizes na compreensão de informações apresentadas oralmente a fim de complementá-las.

Outro benefício que os vídeos podem oferecer, além de apresentar a linguagemalvo por meio de input visual e verbal, é que os vídeos podem ser extremamente motivadores para os aprendizes. Deci e Ryan (1985) propuseram que a motivação pode ser intrínseca, na qual o aprendiz é movido por fatores internos, como o interesse, relevância para si, expectativa ou satisfação (CROOKES; SCHMIDT, 1991), ou extrínseca, sendo o aprendiz movido por fatores externos, como uma boa classificação, ou para obtenção de nota. Além disso, os aprendizes podem sentir-se motivados a buscar informações além das apresentadas em sala de aula, como pesquisar conteúdos no site YouTube para aprofundar os conceitos e informações estudadas, tornando-se assim também mais autônomos $^{3}$ no seu processo de construção de conhecimento linguístico.

A apresentação de input contextualizado é outra característica importante que vídeos podem ter no contexto de desenvolvimento de inglês como uma LE. Uma vez que os vídeos apresentam a linguagem-alvo com o uso de recursos visual e verbal, o uso de 'pistas de contextualização' - do inglês contextualization clues - (INNACI; SAM, 2017; TUYEN; HUYEN, 2019), oferece aos aprendizes palavras, ou informações que auxiliam na compreensão das informações apresentadas no vídeo, como o entendimento da organização de estruturas frasais, ou no significado de um vocabulário. A apresentação de input de forma contextualizada pode contribuir para um desenvolvimento mais significativo, facilitando a construção coerente de representações mentais da linguagemalvo sendo apresentada.

Outra relevância importante que os vídeos trazem para o contexto de desenvolvimento de LE é a apresentação de input oral (KRASHEN, 1981, 1982), como por exemplo, os documentários, notícias, tutoriais ou até vídeos caseiros. Quando os aprendizes participam de propostas onde eles não somente são expostos às informações para desenvolver a linguagem-alvo, mas também para compreender as informações sendo transmitidas, eles empregam uma série de processos cognitivos, como atenção, memória, tomada de decisões, entre outros, que alicerçam.

\footnotetext{
${ }^{3}$ Autonomia está relacionada à habilidade que permite ao aprendiz ter controle ao usar um conjunto de táticas, como colocar objetivos, escolher material e tarefas, planejar oportunidades de práticas e monitorar e avaliar todo o progresso (TUMOLO; SANTIBANES, 2011), rumo ao desenvolvimento da LE.
} 
Desta forma, o input torna-se, assim como é descrito por Mayer (2009), um processo, não de acumulação de informação, mas de construção de conhecimento. $O$ processo de construção de conhecimento está relacionado com o fato de os aprendizes construírem representações mentais das informações que estão sendo 'assistidas' - no caso de vídeos - para depois serem organizadas em redes de conhecimento armazenadas na memória de longo prazo, conforme a Teoria Cognitiva da Aprendizagem Multimídia, formulada por Mayer (2005b), e é apresentada na Figura 1.

Figura 1: Teoria Cognitiva da Aprendizagem Multimídia, Baseado em Mayer (2005a).

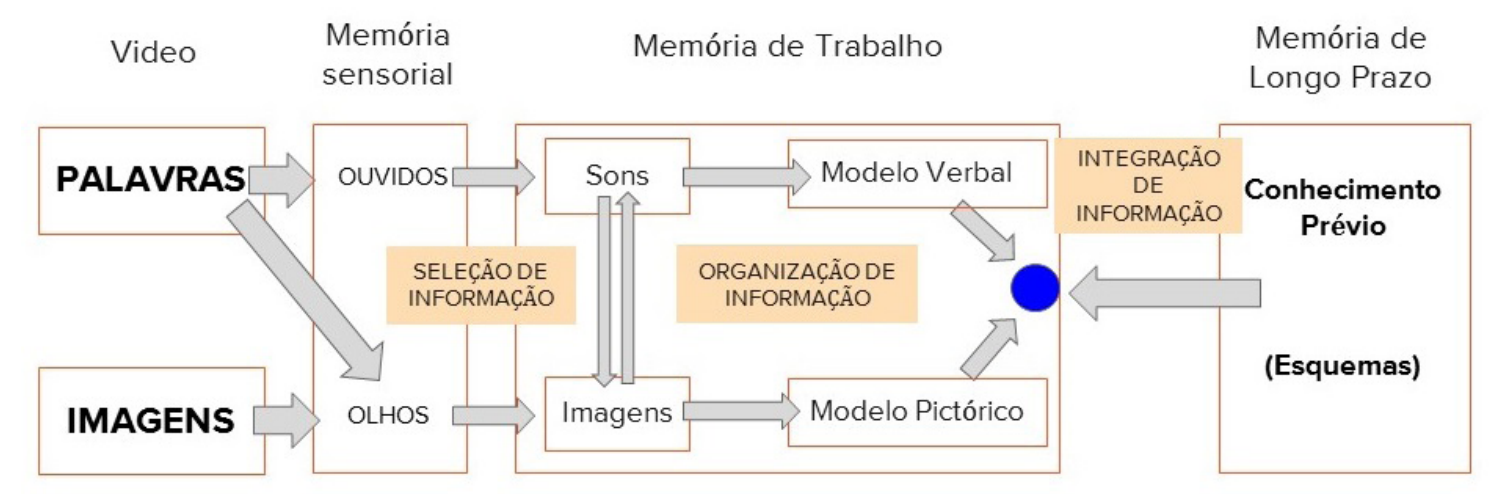

Como pode ser observado na figura apresentada acima, para Mayer (2005a), as informações visuais e verbais entram no sistema cognitivo do aprendiz pela memória sensorial, ou seja, pelos olhos e pelos ouvidos, onde passam por um processo de seleção. Isso implica que o aprendiz esteja envolvido no seu processo de aprendizagem pois seleciona as informações, ou partes delas que julga serem importantes para a sua compreensão. Após a seleção de informações, elas são conduzidas para a memória de trabalho, onde passam por um processo no qual as imagens são convertidas em sons, e os sons em imagens (e.g. a palavra car é convertida em uma imagem de um carro, e a imagem de uma casa é convertida na palavra house), ou seja, os aprendizes constroem representações mentais do input recebido. Após as informações verbais e visuais serem convertidas, elas são organizadas em um modelo verbal e visual. É importante observar também que a memória de longo prazo tem fundamental importância no processo de construção das representações mentais das informações recebidas. Neste processo, o Revista X, v. 16, n. 3, p. 728-741, 2021. 
conhecimento prévio - ou esquemas - já armazenado na memória de longo prazo tem função de firmar a construção do conhecimento, uma vez que as informações que já estão armazenadas na memória de longo prazo do aprendiz são trazidas de volta para a memória de trabalho para serem reprocessadas com as informações novas e, assim, serem integradas como um todo novamente na memória de longo prazo.

Ademais, quando vídeos são agregados ao contexto de ensino/desenvolvimento de inglês como LE, os aprendizes têm a oportunidade de desenvolver estratégias de escuta. De acordo com Thompson e Rubin (1996, apud STANLEY, 2013, p. 81), o processo de ouvir, ao invés de passivo, é um processo ativo, no qual os aprendizes ouvem, selecionam e interpretam a informação a fim de compreendê-la. Este fator pode ser claramente relacionado à Teoria Cognitiva de Aprendizagem Multimídia, uma vez que, quando expostos a vídeos, os aprendizes assumem o papel de construtores ativos de conhecimento, selecionando as partes da informação importantes, a fim de que ela seja manipulada e reorganizada em blocos mentais, resultando na incorporação da mesma (MAYER, 2009).

Considerando o fato que vídeos envolvem o uso de imagens e palavras (nas formas oral e escrita), o processo de desenvolvimento da língua-alvo é beneficiada. Com base nos argumentos trazidos por Mayer (2005a; 2009), é possível dizer que este tipo de recurso educacional deve ser utilizado de maneiras diversas para diferentes objetivos quando relacionadas ao desenvolvimento de uma LE. O processo de desenvolvimento de uma LE pode ser altamente beneficiado com o uso de materiais audiovisuais, uma vez que com estes, os aprendizes experimentam a língua-alvo em diferentes combinações de input (PLASS; JONES, 2005), como é o caso da inserção de vídeos na abordagem baseada em tarefas (do inglês: Task-Based Language Teaching, ou TBLT) (ELLIS, 2003; NUNAN, 2004; WILLIS; WILLIS, 2007). Além do input, há também oportunidades de produzir output, ou seja, produzir a linguagem sendo estudada e desenvolvida, ou também como a própria produção de vídeos, onde os aprendizes se tornam autores de suas produções e utilizam da língua-alvo para transmitir informações (que será discutido mais adiante).

\section{RECURSOS MULTIMIDIÁTICOS PARA O DESENVOLVIMENTO DE IN- GLÊS COMO LÍNGUA ESTRANGEIRA}

Como a tecnologia digital tornou-se parte integral das vidas das pessoas (CHAPELLE; SAURO, 2017), uma das questões principais é como melhor integrá-la no processo de ensinar e desenvolver uma LE, em contexto pedagógico, e o mais importante, 
como fazer esta combinação se tornar mais eficaz e significativa (CHAPELLE; SAURO, 2017). Stanley (2013) defende que a tecnologia digital deveria fazer parte do currículo das escolas, uma vez que ela pode ser usada para apresentar exemplos dos conteúdos sendo estudados, assim como ajudar os aprendizes a se tornarem autônomos. Por exemplo, a criação de comunidades de aprendizagem como blogs, ou até mais recentemente canais no site YouTube para compartilhar informações e não apenas recebê-las, tornando os aprendizes ativos na criação e compartilhamento do conhecimento com os outros.

O uso da tecnologia digital pode ser explorado por professores e instrutores para que o desenvolvimento aconteça de forma mais significativa e contextualizada. No processo de desenvolvimento de uma LE, podemos defender que é indispensável que a tecnologia digital seja parte da rotina da sala de aula, não como algo a ser usado como 'um escape' da rotina da aula, mas como algo que é parte de todo o processo de desenvolvimento.

Além de vídeos, materiais multimídia que podem ser utilizados para fins pedagógicos são os jogos digitais e os inúmeros sítios disponíveis online, onde uma gama infinita de informações pode ser encontrada. Ma (2017), por exemplo, discute extensivamente diferentes alternativas que podem ser oferecidas aos aprendizes no processo de aquisição de vocabulário em ambientes online. Para isto, a autora exemplifica com sistemas computacionais que focam em atividades de aquisição de vocabulário, ou websites que apresentam aos aprendizes o vocabulário em formas de cartões (flashcards) que mostram uma imagem e o nome por escrito. Ao utilizar tais ferramentas, os aprendizes tornam-se mais motivados para desenvolver a língua alvo através de um novo recurso digital.

Conforme mencionado por Gee (2009), com o novo século, novas demandas educacionais surgiram - incluindo o uso de tecnologias digitais e recursos multimidiáticos. Estas novas demandas unem a tecnologia digital com propósitos pedagógicos, como por exemplo: pensamento crítico, resolução de problemas, tomada de decisões, autonomia, trabalho em equipe e colaborativo, liderança, empatia, agência, criatividade, dentre outros (GEE, 2009). Levando isso em consideração, projetos podem ser aplicados nas práticas didático-pedagógicas para o desenvolvimento de inglês como língua estrangeira incluindo o uso de vídeos, de forma a suprir estas demandas. Alguns exemplos são projetos como: a) vídeo onde os aprendizes produzem tutoriais ensinando como criar ou recriar algo, como uma receita, um origami ou alguma peça de artesanato - usando a língua estrangeira como meio de comunicação para apresentação; b) vídeos com ilustrações das letras de músicas na língua estrangeira, a fim de desenvolver diversas 
estruturas da língua, como, por exemplo, os tempos verbais ou vocabulário específico; e c) vídeos produzidos a partir de peças teatrais ou livros, criando um festival de filmes a fim de que aprendizes possam praticar a língua estrangeira de forma comunicativa e contextualizada, entre outros.

Exemplos como estes projetos acima mencionados envolvem 1) o uso de recursos multimidiáticos, como vídeos, para fins educativos; 2) o uso da língua estrangeira para aprendizado contextualizado; 3 ) as novas demandas do século que trabalham de forma a integrar os conhecimentos e experiências anteriores dos discentes aos conhecimentos curriculares; e 4) a autonomia e agência dos aprendizes ao trazer para o contexto educacional recursos digitais que são comumente utilizados por eles. Desta forma, ao trazer para o ambiente educacional os recursos digitais para motivar e facilitar o ensino e o desenvolvimento da LE, o aprender e o fazer tornam-se inseparáveis, a tecnologia digital é incluída em todo o processo, promovendo o desenvolvimento além do plano de ensino curricular, possibilitando o crescimento do aprendiz de forma integral.

\section{HISTÓRIAS DIGITAIS COMO RECURSO DIGITAL PARAAQUISIÇÃO DE PRONÚNCIA E ESCRITA EM INGLÊS COMO LÍNGUA ESTRANGEIRA}

Histórias digitais (doravante HDs) também são recursos multimidiáticos que podem auxiliar no desenvolvimento de uma língua estrangeira. De acordo com Tumolo (2015; 2018), HDs podem ser definidas como um recurso digital contendo alguma mistura de imagens digitais, texto, narração oral gravada, videoclipes e/ou música, com tempo de duração entre 2 e 10 minutos. As HDs podem conter tópicos variados, que vão desde histórias pessoais a eventos históricos, e devem ser narradas por quem as produziu.

Para desenvolver uma HD, Jake (2009) propõe alguns passos a serem seguidos a fim de melhor organizar o desenvolvimento do trabalho. O primeiro deles é a definição do tema, onde o autor da HD a ser desenvolvida deve escolher o tema a ser abordado. Os temas podem ser variados e geralmente englobam um momento na vida do autor como um aniversário, uma formatura ou qualquer momento especial da vida da pessoa que dará voz a essa história. Em seguida, o script começa a ser desenvolvido e os aprendizes são convidados a produzirem o texto escrito da história que será contada. Considerando o contexto de desenvolvimento de LE, nesse passo os aprendizes também recebem feedback escrito a fim de melhorar o texto produzido.

Ainda de acordo com Jake (2009), assim que o script é concluído, os aprendizes começam a produção do storyboard. É nesse passo que o texto escrito deve ser associado com as gravuras digitais que aparecerão no vídeo a ser produzido. É importante ressaltar Revista X, v. 16, n. 3, p. 728-741, 2021. 
que esse passo vai ajudar o autor a realmente refletir sobre as imagens escolhidas analisando se as mesmas estão de fato contribuindo para a construção da história que se pretende contar. O último passo é a utilização de um software para produzir o vídeo com a narração oral do texto previamente desenvolvido no script.

Tendo em vista os passos supracitados, necessários para a produção de uma HD, podemos afirmar que HDs têm o potencial de auxiliar no desenvolvimento das quatro habilidades em uma língua estrangeira. Por meio do desenvolvimento do script, por exemplo, é possível desenvolver as habilidades de leitura e escrita e, por meio da narração, as habilidades de fala e escuta. Neste sentido, o desenvolvimento da leitura pode ser ampliado se usado para a pesquisa do conteúdo que será trabalhado: os aprendizes terão de ler e pesquisar muito antes de escrever um roteiro, terão de ler e aprender o passo a passo, enredo, cenários, entre outros. Por outro lado, o desenvolvimento da escrita será ampliado e utilizado para a criação do script e storyboard, da estruturação dos personagens, se for o caso, entre outros (TUMOLO, 2015).

A pronúncia na LE pode ser desenvolvida, também, durante o processo de gravação da narração oral para a HD. Sendo a pronúncia um dos subcomponentes da fala, ter uma pronúncia inteligível na língua estrangeira é essencial para que haja comunicação. No entanto, o ensino de pronúncia de língua inglesa é conhecido por ser desafiador para a maioria dos aprendizes e professores. Embora tenha sido uma das áreas mais beneficiadas pelos recursos tecnológicos - que vão desde as tecnologias de áudio e vídeo que possibilitaram os aprendizes a terem acesso a amostras de falantes nativos e visualizar os movimentos das articulações necessários para falar na língua estrangeira, até os softwares e aplicativos disponíveis nos computadores e tecnologias móveis atuais - o ensino de pronúncia é muitas vezes negligenciado nas salas de aula e materiais de língua inglesa (SILVEIRA, 2004; STANLEY, 2013). Fatores como a falta de conhecimento específico e insegurança de professores em relação à pronúncia, o tempo limitado em sala de aula, e o grande número de aprendizes contribuem para o ensino de pronúncia permanecer negligenciado (ALBINI; KLUGE, 2013; HARMER, 2007).

Por estes motivos, é importante incentivar os aprendizes a praticarem a pronúncia dentro e fora de sala de aula (BRINTON, 2018; STANLEY; 2013), o que tem se tornado mais fácil tendo em vista a disponibilidade de recursos digitais e, especialmente, das tecnologias móveis, que atualmente fazem parte do dia a dia dos estudantes. Deste modo, tecnologias móveis como smartphones e tablets podem ser utilizados para desenvolver uma HD tanto dentro de sala de aula como fora dela. 
De acordo com Celce-Murcia et al. (2010), o ensino de pronúncia deve ser feito por meio de uma abordagem comunicativa, englobando não apenas a repetição de sons e frases preestabelecidas, mas permitindo que os aprendizes também se comuniquem na língua alvo fazendo uso tanto de aspectos segmentais (sons) quanto suprassegmentais (tonicidade de palavras e frases, ritmo, etc.) da língua estrangeira. Sendo as HDs um meio de comunicação por meio do qual os aprendizes apresentam informações, conceitos, e ideias para uma audiência, de maneira planejada, elas têm potencial para auxiliar no desenvolvimento da pronúncia de maneira comunicativa.

O processo de gravação da narração das HDs também possibilita os aprendizes a ouvirem sua produção e tomar consciência de sua pronúncia na língua inglesa, muitas vezes decidindo por editar e repetir este processo até se sentirem satisfeitos com o resultado. De acordo com Celce-Murcia et al. (2010), o feedback é um elemento essencial para o desenvolvimento da pronúncia, podendo ser ele feito pelo próprio aprendiz, por um colega, ou pelo professor. Sabendo das limitações relacionadas às salas de aula numerosas e tempo limitado, o fato de o próprio aprendiz poder avaliar sua produção durante o desenvolvimento de uma HD pode contribuir para o desenvolvimento da pronúncia.

Estudos ainda revelam que antes de produzirem uma HD, alguns aprendizes nunca tinham tido a oportunidade de se ouvir na língua estrangeira, e que por meio delas, muitos puderam tomar consciência das dificuldades relacionadas à pronúncia que tinham na língua alvo e que deveriam ser melhoradas para que a mensagem da HD pudesse não apenas ser transmitida com sucesso, mas também emocionar quem estava a assisti-las (CASTAÑEDA, 2013).

Outrossim, além da pronúncia, o processo de criação de uma HD pode contribuir positivamente para o desenvolvimento da habilidade da escrita dos aprendizes de uma língua estrangeira. Como mencionado anteriormente, durante a produção do script, os aprendizes recebem feedback escrito dos professores com a finalidade de revisar o texto e deixá-lo ainda melhor. Um estudo desenvolvido com graduandos do curso de Letras Inglês da Universidade Federal de Santa Catarina (UFSC) mostrou que o feedback escrito recebido durante o desenvolvimento do script para a produção de uma HD contribuiu para o desenvolvimento do script e, consequentemente, para o desenvolvimento da habilidade escrita, de acordo com a percepção dos mesmos (ROCHA, 2019).

Os participantes destacaram a contribuição positiva do feedback durante a produção do script. Um dos aspectos enfatizados por eles foi a questão da organização textual, pois através das sugestões recebidas no feedback, eles puderam organizar de forma mais clara as ideias a serem apresentadas durante a HD. Um outro aspecto 
destacado pelos participantes foi o desenvolvimento da percepção deles a respeito das diferenças entre os textos acadêmicos que eles produziam para as disciplinas do curso de Letras e do texto produzido no script para a HD. O texto para a HD não necessitava de uma linguagem tão elaborada quanto os textos acadêmicos e alguns participantes relataram que após o feedback, reescreveram algumas partes de seus scripts a fim de utilizar uma linguagem mais simples capaz de atingir um maior público (ROCHA, 2019).

Ao fazer uso de projetos para a criação de HDs como auxiliar no processo de desenvolvimento de LE, muitas demandas do novo século (GEE, 2009) podem ser supridas. Cumprindo o passo a passo para a criação de HDs, como a escolha do tema, do contexto, da relevância pessoal, da elaboração do script e do storyboard, da gravação e levando em consideração o feedback do professor, o aprendiz desenvolve outras habilidades. Além do aprendizado e do aperfeiçoamento de pronúncia e da escrita na língua-alvo, há ainda o desenvolvimento da autonomia, da agência, da criatividade, da tomada de decisões, do pensamento crítico, entre outros. Portanto, recursos digitais como as HDs podem ser aplicados em sala de aula para aprendizados múltiplos.

\section{CONSIDERAÇÕES FINAIS}

Muitos são os recursos digitais disponíveis atualmente para serem utilizados no contexto de ensino e desenvolvimento de línguas estrangeiras. Neste texto, apresentamos alguns que podem ser usados para o desenvolvimento de uma LE, a saber, o vídeo como recurso multimidiático para a aquisição de inglês como LE, e a história digital, cujas etapas de produção permitem o desenvolvimento de todas as quatro habilidades da língua inglesa. Os recursos disponíveis, juntamente com as estratégias adequadas quando incorporadas em projetos, ou tarefas, onde o foco principal é a comunicação e expressão de ideias e sentimentos dos aprendizes - permitem o desenvolvimento da língua estrangeira alvo. Esse desenvolvimento, assim, conta com uma variedade de recursos digitais e de estratégias pedagógicas, oferecendo aos aprendizes variadas oportunidades não somente para desenvolver a língua-alvo, mas também para se tornarem sujeitos ativos no seu processo de construção de conhecimento.

\section{AGRADECIMENTOS}

Agradecemos nossa colega Luana Garbin Baldissera que participou da revisão inicial informal deste artigo a pedido dos autores. 


\section{REFERÊNCIAS}

ALBINI, A.; KLUGE, D. C. Professores de inglês da rede pública paranaense e o ensino da pronúncia. Revista de Letras DACEX/UTFPR, v. 2011, n. 1, p. 1-12, 2011.

BRINTON, D. M. Innovations in pronunciation teaching. In KANG, O.; THOMPSON, R. I.; MURPHY, J. M. (Eds.), The Routledge Handbook of English Contemporary Pronunciation. New York: Routledge, 2018. p. 449-461.

CASTAÑEDA, M. "I am proud that I did it and it's a piece of me". Digital Storytelling in the foreign language classroom. Calico Journal, v. 30 n. 1, p. 44-62, 2013.

CELCE-MURCIA, M.; BRINTON, D. M.; GOODWIN, J. M. Teaching Pronunciation: A Reference for Teachers of English to Speakers of Other Languages. 14 ed. New York: Cambridge University Press, 2010.

CHAPELLE, C. A.; SAURO, S. Introduction to the Handbook of Technology and Second Language Teaching and Learning. In CHAPELLE, C. A.; SAURO, S. (Eds.) The Handbook of Technology and Second Language Teaching and Learning. Oxford, UK: Wiley Blackwell, 2017. p. 1-9.

CROOKES, G.; SCHMIDT, R. W. Motivation: Reopening the Research Agenda. Language Learning. v. 41, no 4, p. 469-512, 1991.

DANAN, M. Captioning and Subtitling: Undervalued Language Learning Strategies. Meta. v. 49, no 1, p. 67-77, 2004.

DECI, E. L.; RYAN, R. M. Intrinsic Motivation and Self-Determination in Human Behavior. New York: Plenum, 1985.

ELLIS, R. Corrective Feedback and teacher development. L2 journal, v. 1, n. 1, p. 3-18, 2009.

ELLIS, R. Task-Based Language Learning and Teaching. New York, NY: Oxford, 2003.

GEE, J. P. Games, learning, and 21st Century survival skills. Journal of Virtual Worlds Research, v. 2, n. 1, p. 3-9, 2009.

HARMER, J. The Practice of English Language Teaching. Pearson Longman, 2007.

JAKE, D.S. Capturing stories, capturing lives: An introduction to Digital Stories. Illinois, 2009. 
INNACI, D. L.; SAM, P. Using Context Clues as a Vocabulary Learning Strategy: An Experimental Study. Journal of English and Literature (JOELL). v. 4, n. 3, p. 39-43, 2017.

KRASHEN, S. D. Second language acquisition and second language learning. Oxford: Pergamon, 1981.

KRASHEN, S. D. Principles and Practice in Second Language Acquisition. Oxford: Pergamon, 1982.

LAVAUR, J. M.; BAIRSTOW, D. Languages on the screen: Is film comprehension related to the viewers' fluency level and to the language in the subtitles? International Journal of Psychology. v. 46, nº 6, p. 455-462, 2011.

MA, Q. Technologies for Teaching and Learning Vocabulary. In CHAPELLE, C. A.; SAURO, S. (Eds.) The Handbook of Technology and Second Language Teaching and Learning. Oxford, UK: Wiley Blackwell, 2017. p. 45-61.

MAYER, R. E. Introduction to Multimedia Learning. In (Ed.) The Cambridge Handbook of Multimedia Learning. New York, USA: Cambridge University Press, p. $1-16,2005 \mathrm{a}$.

MAYER, R. E. Cognitive Theory of Multimedia Learning. In (Ed.) The Cambridge Handbook of Multimedia Learning. New York, USA: Cambridge University Press, p. 3148, $2005 b$.

MAYER, R. E. Multimedia Learning. $2^{\text {nd }}$ Ed. New York. Cambridge University Press, 2009.

NUNAN, D. Task-Based Language Teaching. United Kingdom: Cambridge University Press, 2004.

PLASS, J. L; JONES, L. C. Multimedia Learning in Second Language Acquisition. In MAYER, R. E. (Ed.). The Cambridge Handbook of Multimedia Learning. New York: Cambridge University Press, p. 467-488, 2005.

ROCHA, V. Digital Stories: Feedback Contribution During Script Development. Dissertação de Mestrado do Programa de Pós-Graduação em Inglês. Universidade Federal de Santa Catarina, Florianópolis, 2019.

SILVEIRA, R. The Influence of Pronunciation Instruction on the Perception and the Production of English Word-Final Consonants. Tese de Doutorado do Programa de PósGraduação em Inglês. Universidade Federal de Santa Catarina, Florianópolis, 2004. 
STANLEY, G. Language Learning with Technology: Ideas for Integrating Technology in the Classroom. Cambridge, UK: Cambridge University Press, 2013.

THOMPSON, I.; RUBIN, J. Can Strategy Instruction Improve Listening Comprehension?, Foreign Language Annals, v. 29, n. 3, p. 331-342, 1996.

TUMOLO, C. H. S.; SANTIBANEZ, V. S. Autonomia em graduação na modalidade a distancia: estratégias de organização e de aprendizagem. In: IX Congresso Brasileiro de Linguística Aplicada - Linguística Aplicada \& Sociedade, 2012, Rio de Janeiro. Anais Eletrônicos IX Congresso Brasileiro de Linguística Aplicada, 2011. v. 1.

TUMOLO, C. H. S. Recursos digitais e aprendizagem de inglês como língua estrangeira. Ilha do Desterro, n. 66, p. 203-238, 2014.

TUMOLO, C. H. S. Recursos digitais para ensino/aprendizagem de inglês como língua estrangeira: o vídeo em destaque. In TOMITCH, L. M. B.; HEBERLE, V. M. (Orgs.), Perspectivas Atuais de Aprendizagem e Ensino de Linguas. Florianópolis: LLE/CCE/ UFSC, 2017. p. 51-75.

TUMOLO, C. H. S.. Histórias Digitais como Recurso para Ensino/Aprendizagem de Inglês como Língua Estrangeira. Estudos Anglo-Americanos, v. 1, p. 101-117, 2015.

TUMOLO, C. H. S. Digital Stories for L2 and their development process. Revista Horizontes de Linguística Aplicada, 17, n. 1, p. 15-30, 2018.

TUYEN, L. V.; HUYEN, V. TN. Effects of using Contextual Clues on English Vocabulary Retention and Reading Comprehension. International Journal of English, Literature and Social Science (IJELS). v. 4, nº 5, p. 1342-1347, 2019.

WILLIS, D.; WILLIS, J. Doing Task-based Teaching. New York, NY: Oxford, 2007.

Recebido em: 22 fev. 2021.

Aceito em: 21 abr. 2021. 